\title{
Tendência de atitudes extremas em relação ao peso em adolescentes e sua relação com suporte familiar e imagem corporal
}

\author{
Tendency of extreme attitudes in relation to weight in adolescents \\ and their relationship with family support and body image
}

Carolina Souza Ferreira (https://orcid.org/0000-0002-7600-6690) ${ }^{1}$

Fabíola Bof de Andrade (https://orcid.org/0000-0002-3467-3989) ${ }^{1}$

${ }^{1}$ Centro de Pesquisas René Rachou, Fundação Oswaldo Cruz. Av. Augusto de Lima 1715, Barro Preto. 30190-002 Belo Horizonte MG Brasil.fabiola.bof@ fiocruz.br

\begin{abstract}
The scope of this study was to evaluate the tendency of extreme attitudes in relation to body weight among adolescents in Brazilian capitals and to verify its relationship with measures of family support and perception of body image. A cross-sectional study was conducted based on data from the National School Health Survey (PeNSE) for the years 2009, 2012 and 2015. There was an increase in the prevalence of extreme attitudes during the period evaluated. In the final model, higher age, perception of body image as being fat and male gender were associated with a higher incidence of extreme attitudes. However, higher level of schooling of the mother and the variables associated with family support (living with parents, informed parents, eating with parents) were associated with a lower incidence of extreme attitudes. The results of this study revealed that the family and social context is a fundamental issue to be investigated with families, adolescents and schools, as a preventive measure for possible health problems. The need to restructure public policies on health and education for adolescents, which should have the encouragement of family support as a guideline, is suggested.
\end{abstract}

Key words Adolescent, Body image, Body weight, School health services, Vomiting
Resumo O objetivo deste estudo foi avaliar a tendência de atitudes extremas em relação ao peso corporal entre adolescentes das capitais brasileiras e verificar a sua relação com medidas de suporte familiar e percepção da imagem corporal. Realizou-se um estudo transversal com base nos dados da Pesquisa Nacional de Saúde do Escolar (PeNSE) dos anos de 2009, 2012 e 2015. Observou-se aumento da prevalência de atitudes extremas no periodo avaliado. No modelo final, maior idade, percepção da imagem corporal como gordo e sexo masculino foram associados a maiores chances de atitudes extremas. Já maior escolaridade da mãe e as variáveis associadas a suporte familiar (mora com os pais, responsáveis informados e refeição com responsáveis) foram associadas a menores chances de atitudes extremas. Os resultados do presente estudo evidenciaram que o contexto familiar e social é um assunto fundamental para ser trabalhado com as famílias, os adolescentes $e$ as escolas, como medida de prevenção a possíveis problemas de saúde. Sugere-se a necessidade de reestruturação das políticas públicas de saúde e educação voltadas aos adolescentes, que devem ter como diretrizes o incentivo ao apoio familiar.

Palavras-chave Adolescente, Imagem corporal, Peso corporal, Serviços de saúde escolar, Vômito 


\section{Introdução}

A adolescência é o período da vida no qual ocorrem intensas transformações físicas, psicológicas e comportamentais ${ }^{1}$. Neste período de transição entre a infância e a idade adulta, os jovens podem enfrentar problemas de saúde mental, como depressão e transtornos alimentares ${ }^{2}$. A imagem corporal é uma construção psicológica que faz parte da autoimagem. Sua importância aumenta à medida que os jovens se tornam mais conscientes do corpo com as mudanças físicas associadas à puberdade ${ }^{3}$. Nas últimas décadas, ocorreu aumento da prevalência de excesso de peso no mundo $\mathrm{o}^{4}$ e as preocupações e insatisfação com a imagem corporal aumentaram ${ }^{5}$. Estudos apontaram maior prevalência de insatisfação com a imagem corporal entre adolescentes com excesso de peso $^{5-7}$.

Comportamentos de controle de peso resultantes da insatisfação com a imagem corporal incluem práticas prejudiciais a saúde ${ }^{8}$, como provocar vômito e tomar remédios para emagrecer ${ }^{9,10}$. Estudo realizado com adolescentes noruegueses verificou que os comportamentos de controle de peso não saudáveis foram significativamente associados com maior Índice de Massa Corporal, autopercepção do excesso de peso, baixa autoestima e depressão. As estimativas de prevalência para o uso de laxantes permaneceram relativamente constantes nas três ondas de pesquisa; já o uso de pílula dietética apresentou aumento significativo com o tempo ${ }^{11}$. No Brasil, Claro et al. ${ }^{12}$ encontraram entre adolescentes prevalências de $6,1 \%$ e $5,7 \%$ de uso de laxantes ou vômito induzido e de uso de medicamentos para perda de peso, respectivamente; em estudo mais recente, estas prevalências aumentaram para $7,4 \%$ e $6,7 \%$, respectivamente ${ }^{13}$. A aceitação pelos pares e pela família e boas relações sociais são fatores de proteção contra o excesso de preocupação com a imagem corporal ${ }^{14}$. Estudos mostraram que a maior frequência de refeições familiares está associada a menores chances do desenvolvimento de transtornos alimentares, sentimentos de depressão ${ }^{15}$ e de adoção de comportamentos não saudáveis para o controle de peso ${ }^{16}$.

Tendo em vista a importância do apoio familiar em relação à manutenção de um peso saudável e para a aceitação corporal, o presente trabalho tem como objetivo avaliar a tendência de atitudes extremas em relação ao peso corporal entre adolescentes brasileiros e verificar a sua relação com medidas de suporte familiar e percepção da imagem corporal nos anos de 2009, 2012 e 2015.

\section{Métodos}

Foi realizado um estudo transversal com dados da Pesquisa Nacional de Saúde do Escolar (PeNSE) realizada nos anos 2009, 2012 e 2015. A amostra da PeNSE é representativa dos estudantes do $9^{\circ}$ ano do Ensino Fundamental de escolas públicas e privadas nas 26 capitais brasileiras e no Distrito Federal. Todos os detalhes da pesquisa e do processo de amostragem foram publicados previamente ${ }^{17,18}$. Neste estudo, foram analisados 55.670 estudantes em 2009, 60.496 em 2012 e 50.302 em 2015.

A variável dependente foi a ocorrência de alguma atitude extrema em relação ao peso (sim, não). Esta foi avaliada por meio do autorrelato do uso de laxante ou indução de vômito ("Nos últimos 30 dias, você vomitou ou tomou laxantes para perder peso ou evitar ganhar peso? sim/ não") ou do uso de medicamento ou fórmula para perda de peso ("Nos últimos 30 dias, você tomou algum remédio, fórmula ou outro produto para perder ou manter seu peso sem acompanhamento médico? sim/não"). Indivíduos que relataram pelo menos uma das duas práticas foram classificados como com atitude extrema. As perguntas foram as mesmas nos três anos da pesquisa.

As variáveis independentes de interesse foram a imagem corporal e medidas de suporte familiar. A imagem corporal foi investigada por meio da seguinte pergunta: "Quanto ao seu corpo, você se considera: muito magro(a), magro(a), normal, gordo e muito gordo(a)?". Neste estudo, os escolares foram classificados em três níveis: magro (incluindo magro e muito magro), normal e gordo (incluindo gordo e muito gordo). Foram consideradas três variáveis de suporte familiar: mora com os pais (não, somente com a mãe, somente com o pai, mora com mãe e pai); responsáveis informados (não [nunca/raramente/às vezes], sim [a maioria do tempo/sempre]); refeição com responsáveis (não [3 a 4 dias por semana/1 a 2 dias por semana/raramente/não], sim [sim, todos os dias/sim, 5 a 6 dias por semana]). A avaliação sobre o grau de informação dos responsáveis sobre o adolescente foi feita pela seguinte pergunta: Nos últimos 30 dias, com que frequência seus pais ou responsáveis sabiam realmente o que você estava fazendo em seu tempo livre? A frequência de refeições com os responsáveis foi avaliada a partir da pergunta: Você costuma almoçar ou jantar com sua mãe, pai ou responsável?

Outras covariáveis de ajuste foram: escolaridade da mãe (não estudou, fundamental in- 
completo, fundamental completo/ensino médio incompleto, ensino médio completo/ensino superior incompleto, ensino superior completo, não sabe); sexo (masculino, feminino) e idade (11 a 13 anos, 14 anos, 15 anos, 16 a 19 anos).

Os bancos de dados da PeNSE foram compatibilizados e apensados para sua análise. Inicialmente, foi realizada a descrição das variáveis de estudo em cada ano de realização da pesquisa. Em seguida, realizou-se análise bivariada entre o desfecho e cada uma das variáveis independentes por meio de regressão logística simples seguida de análise multivariada por meio de modelo de regressão logística multivariado. As variáveis foram incluídas no modelo multivariado de forma hierarquizada na seguinte ordem: condições sociodemográficas; medidas de suporte familiar; imagem corporal e ano de estudo. Foram mantidas no modelo todas as variáveis que se mantiveram associadas ao desfecho. As prevalências ajustadas de atitudes extremas foram calculadas a partir do modelo final. As estimativas dos modelos foram interpretadas por meio de odds ratio e respectivos intervalos de confiança de $95 \%$. A partir do modelo de regressão logística multivariado foram calculadas as probabilidades de atitudes extremas em relação ao peso para cada um dos anos da PeNSE e de acordo com as medidas de suporte familiar e satisfação com o peso corporal. Todas as análises foram feitas no programa Stata 14.0 (Stata Corporation, College Station, TX, USA), utilizando-se o comando survey, que permite considerar a estrutura complexa do processo amostral. Todas as análises foram ponderadas pelos pesos amostrais.

O projeto da PeNSE foi aprovado pela Comissão Nacional de Ética em Pesquisa - Conep, do Conselho Nacional de Saúde - CNS, conforme Parecer de Emenda no 005 de 10 de junho de 200919; Parecer no 192/2012, referente ao registro $\mathrm{n}^{\mathrm{o}} 16.805$ do Conep/MS em 27/03/2012 $2^{20}$; e Parecer Conep n. 1.006.467, de 30/03/2015 ${ }^{21}$. Todos os participantes assinaram o Termo de Consentimento Livre e Esclarecido no momento da entrevista.

\section{Resultados}

A Tabela 1 apresenta a distribuição das variáveis independentes de acordo com os anos de estudo. Todas as variáveis apresentaram diferença estatisticamente significativa ao longo dos anos. A prevalência de atitudes extremas aumentou ao longo dos três inquéritos da PeNSE, sendo as prevalências ajustadas iguais a 6,4\% (IC 95\%: 6,0-6,8) em
2009, 9,0\% (IC 95\%: 8,6-9,5) em 2012 e 10,1\% (IC 95\%: 9,5-10,6) em 2015.

A Tabela 2 apresenta a análise bivariada para a associação entre a presença de atitudes extremas em relação ao peso e as variáveis independentes. Observou-se que a chance de atitudes extremas foi significativamente relacionada a todas as variáveis independentes. Também houve aumento da chance de atitudes extremas entre os anos de estudo, verificado pelo aumento da OR no período de 2012 a 2015 em relação a 2009.

A Tabela 3 apresenta o resultado do modelo de regressão logística multivariado para atitudes extremas. A presença de suporte familiar de todas as fontes foi associada a menores chances de atitudes extremas. A chance de atitude extrema foi $18 \%$ menor para escolares que moravam com os pais, $52 \%$ menor para indivíduos que mantinham os responsáveis informados e $10 \%$ menor para os adolescentes que possuíam o hábito de comer com os responsáveis. Adolescentes do sexo feminino apresentaram chance $17 \%$ menor de atitudes extremas em relação aos do masculino. A chance de atitudes extremas também foi menor entre os adolescentes cujas mães apresentavam maior nível de escolaridade quando comparados àqueles cujas mães nunca estudaram. Por outro lado, adolescentes que se perceberam gordos apresentaram chances $142 \%$ maiores de atitudes extremas do que aqueles que perceberam o seu peso como normal. As chances de atitudes extremas foram maiores nos anos de 2012 e 2015 quando comparadas ao ano de 2009.

Pela Figura 1, calculada a partir dos resultados do modelo multivariado, verifica-se que os adolescentes que moravam com os pais, que mantinham os responsáveis informados, que faziam refeições com estes e que apresentavam uma percepção de imagem corporal normal apresentaram menores probabilidades de atitudes extremas em todos os anos de realização da pesquisa.

\section{Discussão}

O presente trabalho avaliou a tendência de atitudes extremas em relação ao peso entre adolescentes das capitais brasileiras e verificou a sua relação com medidas de suporte familiar e percepção da imagem corporal. As prevalências de atitudes extremas em relação ao peso encontradas no presente estudo foram mais elevadas do que aquelas observadas entre adolescentes espanhóis e norte -americanos, 3,26\% e 5,03\%, respectivamente ${ }^{22}$. Observou-se aumento da prevalência de atitudes 


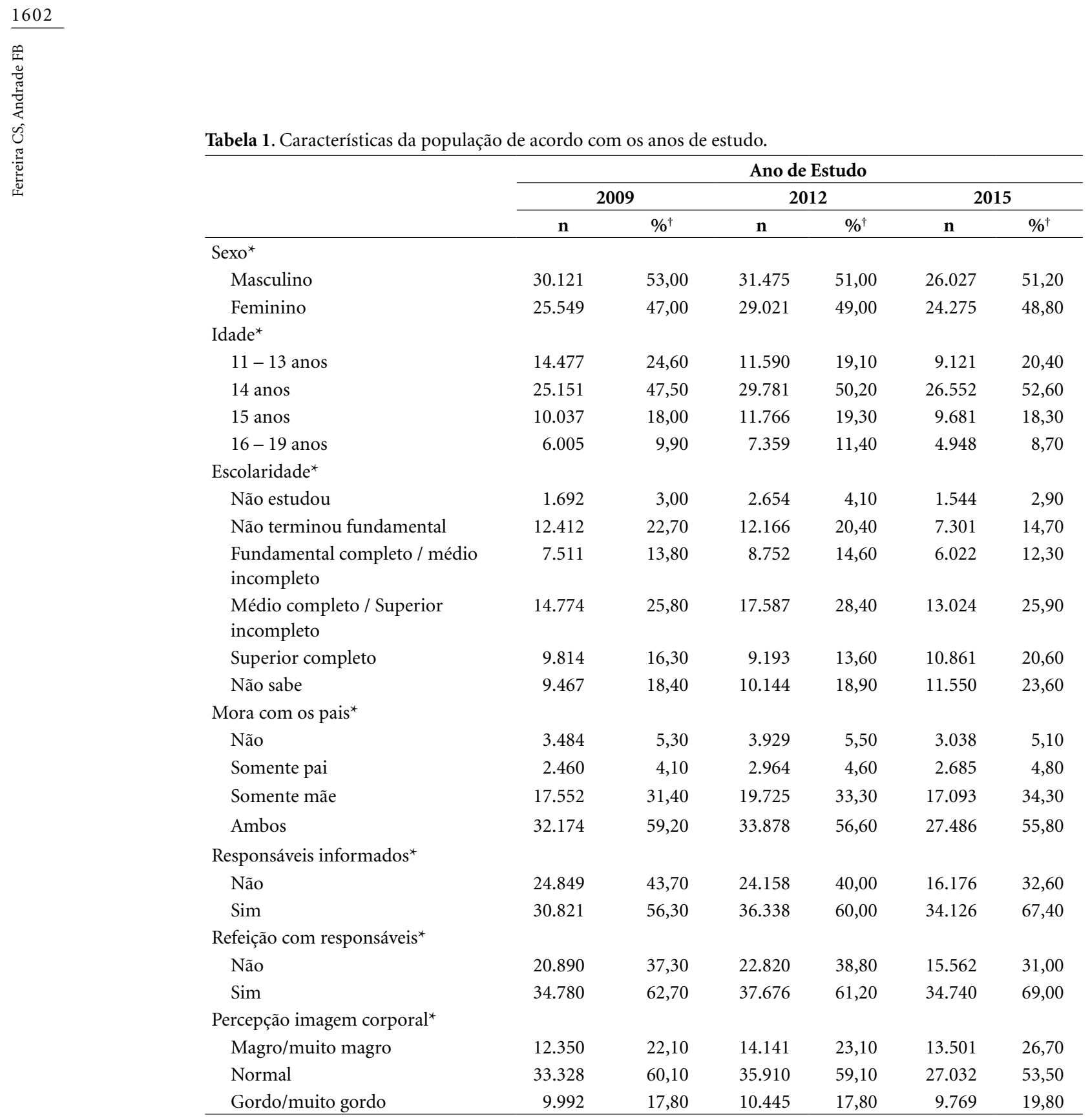

${ }^{\star} \mathrm{p}<0.001 .{ }^{\dagger}$ Análise ponderada.

extremas no período avaliado, o que corrobora com os achados de estudos realizados na Norue$\mathrm{ga}^{11}$ e nos Estados Unidos ${ }^{23}$.

Aspectos da vida familiar, como conversar um com o outro, jantar com companhia e saber sobre os amigos influenciam positivamente em vários indicadores do bem-estar do adolescen$\mathrm{te}^{24}$. Assim, os achados deste estudo confirmam a importância do suporte familiar para a prevenção de atitudes extremas em relação ao peso ${ }^{3,14,25}$. Observou-se em todos os anos que, para todos os tipos de suporte familiar, a prevalência de atitudes extremas foi maior entre os adolescentes sem suporte. Semelhante a esses achados, Wang et al. ${ }^{26}$ verificaram que adolescentes que tinham como hábito jantar com os pais apresentaram menor chance de adotar comportamentos não saudáveis para controle de peso. Vander Wa ${ }^{27}$ verificou que adolescentes que apresentaram comunicação difícil com os pais, baixos níveis de apoio escolar por parte dos pais ou bullying frequente tinham maior probabilidade de adotar comportamentos de controle de peso prejudiciais à saude. Estudos prévios também demonstraram que a frequência de refeições familiares foi positivamente associada à ingestão de alimentos saudáveis ${ }^{25,28} \mathrm{e}$ inversa- 
Tabela 2. Análise bivariada para verificação da associação entre a presença de atitudes extremas em relação ao peso e as variáveis independentes.

\begin{tabular}{|c|c|c|}
\hline & \multicolumn{2}{|c|}{$\begin{array}{l}\text { Atitudes } \\
\text { extremas }\end{array}$} \\
\hline & OR & IC $(95 \%)$ \\
\hline \multicolumn{3}{|l|}{ Sexo $($ ref $=$ masculino $)$} \\
\hline Feminino & 0,84 & $(0,79-0,90)$ \\
\hline Idade & 1,27 & $(1,23-1,31)$ \\
\hline \multicolumn{3}{|c|}{ Escolaridade (ref = não estudou) } \\
\hline $\begin{array}{l}\text { Fundamental } \\
\text { incompleto }\end{array}$ & 0,61 & $(0,53-0,71)$ \\
\hline $\begin{array}{l}\text { Fundamental completo/ } \\
\text { Médio incompleto }\end{array}$ & 0,59 & $(0,50-0,69)$ \\
\hline $\begin{array}{l}\text { Médio completo/ } \\
\text { Superior incompleto }\end{array}$ & 0,57 & $(0,49-0,67)$ \\
\hline Superior completo & 0,61 & $(0,52-0,71)$ \\
\hline Não sabe & 0,55 & $(0,47-0,64)$ \\
\hline \multicolumn{3}{|l|}{ Mora com os pais (ref = não) } \\
\hline Somente pai & 0,71 & $(0,59-0,84)$ \\
\hline Somente mãe & 0,76 & $(0,68-0,86)$ \\
\hline Ambos & 0,64 & $(0,57-0,72)$ \\
\hline \multicolumn{3}{|c|}{ Responsáveis informados (ref = não) } \\
\hline Sim & 0,49 & $(0,46-0,52)$ \\
\hline \multicolumn{3}{|c|}{ Refeição com responsáveis (ref = não) } \\
\hline Sim & 0,75 & $(0,70-0,80)$ \\
\hline \multicolumn{3}{|c|}{ Percepção imagem corporal $($ ref = normal $)$} \\
\hline magro/muito magro & 1,13 & $(1,04-1,23)$ \\
\hline gordo/muito gordo & 2,37 & $(2,19-2,56)$ \\
\hline \multicolumn{3}{|l|}{ Ano do estudo $($ ref $=2009)$} \\
\hline 2012 & 1,44 & $(1,32-1,57)$ \\
\hline 2015 & 1,53 & $(1,39-1,68)$ \\
\hline
\end{tabular}

mente associada à insatisfação corporal e preocupação com o peso ${ }^{15}$.

A natureza protetora das refeições em família sugere que o envolvimento em refeições familiares regulares deve ser recomendado para aquelas com crianças e adolescentes ${ }^{16,25,29}$. Entre os mecanismos possíveis para explicar a relação entre atitudes extremas em relação ao peso e ao suporte familiar, a literatura revela que refeições familiares podem diminuir o risco de comportamentos alimentares não saudáveis, incluindo modelagem parental de padrões saudáveis de alimentação ${ }^{28}$, aumentar as oportunidades de conexão com adolescentes por meio de conversas ${ }^{24}$ e monitorar regularmente os comportamentos alimentares e emocionais do adolescente ${ }^{15}$, permitindo a identificação precoce de problemas ${ }^{16}$.

As refeições em família constituem um cenário de importância social significativa na vida de
Tabela 3. Modelo de regressão logística multivariado para os fatores associados a atitudes extremas em relação ao peso.

\begin{tabular}{lrr}
\hline & $\begin{array}{c}\text { Atitudes } \\
\text { extremas }\end{array}$ \\
\cline { 2 - 3 } & OR & IC (95\%) \\
\hline Sexo (ref = masculino) & & \\
$\quad$ Feminino & 0,83 & $0,78-0,89$ \\
Idade & 1,25 & $1,21-1,29$ \\
Escolaridade (ref = não estudou) & & \\
$\quad$ Fundamental incompleto & 0,74 & $0,63-0,86$ \\
$\quad$ Fundamental completo/ & 0,75 & $0,64-0,88$ \\
$\quad$ Médio incompleto & & \\
$\quad$ Médio completo/Superior & 0,77 & $0,65-0,90$ \\
$\quad$ incompleto & & \\
$\quad$ Superior completo & 0,85 & $0,73-1,00$ \\
$\quad$ Não sabe & 0,64 & $0,55-0,75$ \\
Mora com os pais (ref = não) & & \\
$\quad$ Somente pai & 0,79 & $0,66-0,95$ \\
$\quad$ Somente mãe & 0,88 & $0,78-0,99$ \\
$\quad$ Ambos & 0,82 & $0,73-0,92$ \\
Responsáveis informados (ref = não) & \\
$\quad$ Sim & 0,48 & $0,45-0,51$ \\
Refeição com responsáveis (ref = não) & \\
$\quad$ Sim & 0,90 & $0,84-0,96$ \\
Percepção imagem corporal (ref $=$ normal) & \\
$\quad$ magro/muito magro & 1,08 & $0,99-1,17$ \\
$\quad$ gordo/muito gordo & 2,42 & $2,24-2,61$ \\
Ano do estudo (ref=2009) & & \\
$\quad$ 2012 \\
2015 & 1,46 & $1,34-1,60$ \\
\hline Ref = categoria de referência. & 1,66 & $1,51-1,83$ \\
\hline
\end{tabular}

crianças e adolescentes, pois é importante que os pais repassem para seus filhos boas escolhas alimentares, agindo como modelos positivos para protegê-los dos perigos do ambiente obesogênico da vida moderna ${ }^{29}$. No presente trabalho, filhos de mães com maior escolaridade apresentaram menor chance de atitude extrema. Para Scaglioni et al. ${ }^{29}$, as famílias em que os pais têm um melhor nível educacional apresentam maior chance de obter informações sobre alimentação adequada e de consumir alimentos saudáveis. Reforçando a importância do apoio familiar na adoção de hábitos alimentares saudáveis, Yee et al. ${ }^{30}$ verificaram que comportamentos parentais são fortes correlatos de comportamento de consumo alimentar dos filhos, com destaque para três áreas principais na influência parental: orientação/ educação ativa, mediadores psicossociais e influência dos estilos parentais gerais. É importante 


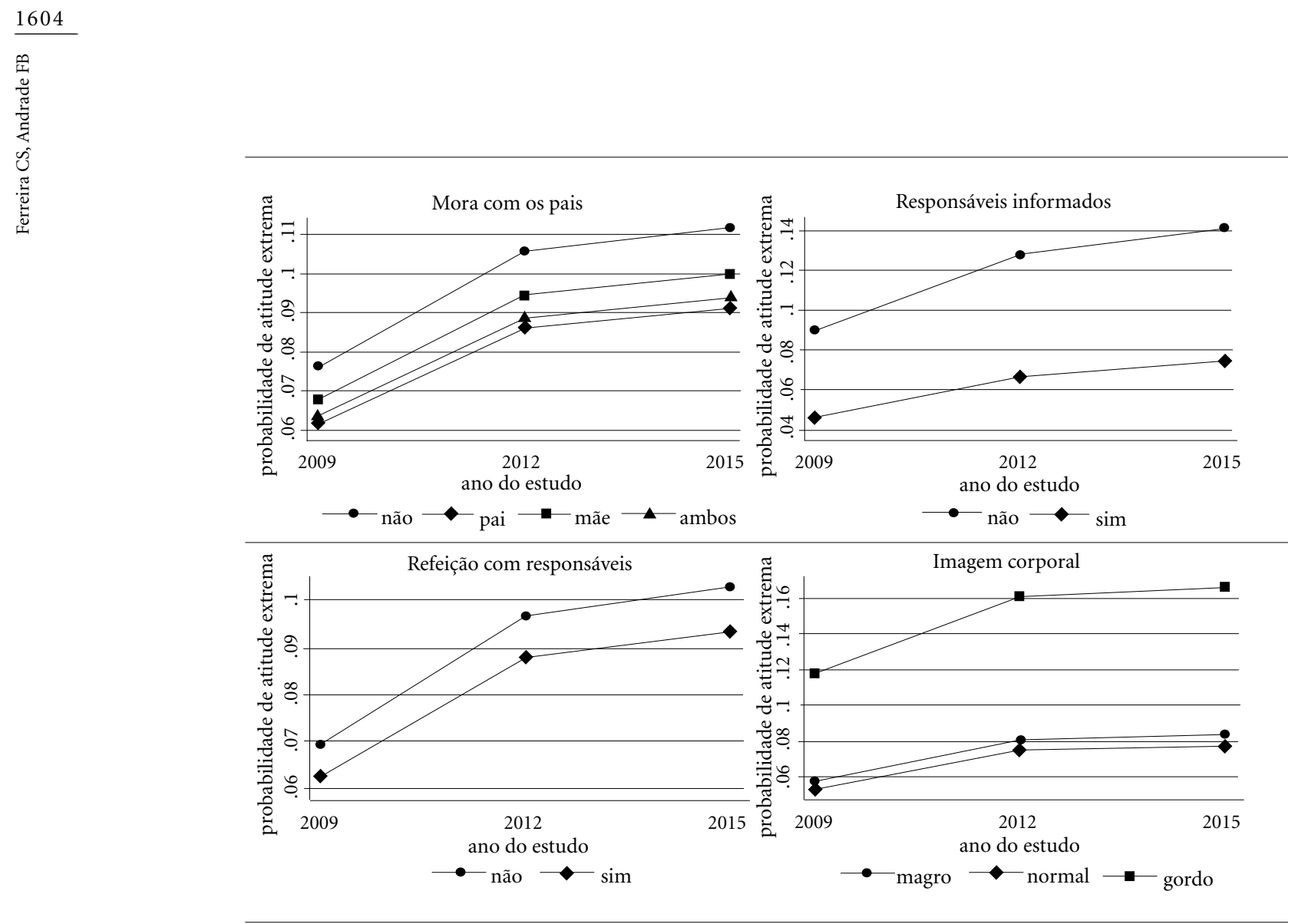

Figura 1. Prevalências de atitudes extremas de acordo com suporte familiar e imagem corporal.

ressaltar que dietas restritivas e atitudes extremas são considerados ineficazes para o controle de peso a longo prazo e estão associados ao ganho de peso e aumento do risco de transtornos alimentares $^{22}$.

O aumento das chances de atitudes extremas em relação ao peso entre os adolesentes que se percebem gordos corroboram achados da literatura $^{5,10,31}$. Sharpe et al. ${ }^{10}$ verificaram que a insatisfação com a imagem corporal foi preditora de transtornos alimentares e comportamentos não saudáveis para controle do peso em adolescentes norte-americanos. De forma semelhante, estudo conduzido com adolescentes do sexo feminino em uma província da África do Sul verificou que a insatisfação com a imagem corporal, a falta de apoio familiar e as opiniões negativas de colegas da mesma idade são fatores de risco para a adoção de práticas não saudáveis para o controle do peso $^{32}$. Esses achados demonstram o impacto negativo de normas sociais relacionadas ao excesso de peso que fazem com que o adolescente adote um comportamento inadequado e não saudável para obter perda de peso. Assim, para evitar a estigmatização do excesso de peso e preocupações excessivas com o mesmo, as famílias, bem como os profissionais da área da saúde e da educação, devem encorajar os adolescentes a adotar hábitos alimentares e comportamentos relacionados com o peso saudáveis, concentrando-se mais no bem estar geral e na promoção da saúde do que exclusivamente na perda de peso $^{31}$.

Este estudo é pioneiro no Brasil em avaliar a tendência de atitudes extremas em relação ao peso entre adolescentes brasileiros e verificar a sua relação com medidas de suporte familiar e percepção da imagem corporal ao longo de um período de seis anos. Contudo, a PeNSE, assim como outros inquéritos de base populacional, apresenta limitações: o uso do autorrelato para a pesquisa de atitudes extremas pode gerar sub -relato, pois o adolescente pode ficar inibido e não contar a verdade Além disso, como os dados incluem apenas adolescentes que frequentam a escola, as prevalências podem não refletir a realidade de toda a população, pois os indivíduos que estão fora do ambiente escolar nas idades avaliadas tendem a ser os mais vulneráveis, propensos a hábitos não saudáveis e com menor rede de apoio familiar ${ }^{33}$. 
Os resultados do presente estudo evidenciaram que o contexto familiar e social é um assunto fundamental para ser trabalhado com as famílias, os adolescentes e as escolas, como medida de prevenção a possíveis problemas de saúde. Assim, os achados apontam para a necessidade de reestruturação das políticas públicas de saúde e educação voltadas aos adolescentes, que devem ter como diretrizes o incentivo ao apoio familiar, bem como a adoção de um ambiente saudável nas escolas. Além disso, o aumento na prevalência de atitudes extremas observado aponta para a necessidade de se dar continuidade a esse inquérito permitindo o monitoramento constante da saúde dos adolescentes brasileiros.

\section{Colaboradores}

CS Ferreira e FB Andrade participaram do planejamento, análise de dados e redação do manuscrito.

\section{Referências}

1. World Health Organization (WHO). Coming of age: adolescent health. Geneva: WHO; 2019.

2. Mairs R, Nicholls D. Assessment and treatment of eating disorders in children and adolescents. Arch Dis Child 2016; 101(12):1168-1175.

3. Currie C, Zanotti CF, Morgan A, Currie DB, de Looze $\mathrm{M}$, Roberts $\mathrm{C}$, et al. Social determinants of health and well-being among young people. Health Behaviour in School-aged Children (HBSC) study: international report from the 2009/2010 survey. Copenhague: WHO Regional Office for Europe; 2012. (Health Policy for Children and Adolescents).

4. Ncd Risk Factor Collaboration (NCD-RisC). Worldwide trends in body-mass index, underweight, overweight, and obesity from 1975 to 2016: a pooled analysis of 2416 population-based measurement studies in 128.9 million children, adolescents, and adults. Lancet 2017; 390(10113):2627-2642.

5. Weinberger NA, Kersting A, Riedel-Heller SG, Luck-Sikorski C. Body Dissatisfaction in Individuals with Obesity Compared to Normal-Weight Individuals: A Systematic Review and Meta-Analysis. Obes Facts 2017; 9(6):424-441.

6. Iepsen AM, Silva MC. Prevalência e fatores associados à insatisfação com a imagem corporal de adolescentes de escolas do Ensino Médio da zona rural da região sul do Rio Grande do Sul, 2012. Epidemiol. Serv. Saúde 2014; 23(2):317-325.

7. Branco LM, Hilário MOE, Cintra IP. Percepção e satisfação corporal em adolescentes e a relação com seu estado nutricional. Rev. psiquiatr. clín. 2006; 33(6):292-296

8. Castro IRR, Levy RB, Cardoso LO, Passos MD, Sardinha LMV, Tavares LF, Dutra SP, Martins A. Imagem corporal, estado nutricional e comportamento com relação ao peso entre adolescentes brasileiros. Cien Saude Colet 2010; 15(Supl. 2):3099-3108. 
9. Del Duca GF, Garcia LMT, Sousa TF, Oliveira ESA, Nahas MV. Insatisfação com o peso corporal e fatores associados em adolescentes. Rev. paul. pediatr. 2010; 28(4):340-346.

10. Sharpe H, Griffiths S, Choo TH, Eisenberg ME, Mitchison D, Wall M, Neumark-Sztainer D. The Relative Importance of Dissatisfaction, Overvaluation and Preoccupation with Weight and Shape for Predicting Onset of Disordered Eating Behaviours and Depressive Symptoms over 15 Years. Int J Eat Disord 2018; 51(10):1168-1175.

11. Stephen EM, Rose JS, Kenney L, Rosselli-Navarra F, Weissman RS. Prevalence and correlates of unhealthy weight control behaviors: findings from the national longitudinal study of adolescent health. J Eat Disord 2014; $2: 16$

12. Claro RM, Santos MAS, Oliveira-Campos M. Imagem corporal e atitudes extremas em relação ao peso em escolares brasileiros (PeNSE 2012). Rev. bras. epidemiol. 2014; 17(Supl. 1):146-157.

13. Silva SU, Barufaldi LA, Andrade SSCA, Santos MAS, Claro RM. Estado nutricional, imagem corporal e associação com comportamentos extremos para controle de peso em adolescentes brasileiros, Pesquisa Nacional de Saúde do Escolar de 2015. Rev. bras. epidemiol. 2018; 21(Supl. 1):e180011.

14. Michael SL, Wentzel K, Elliott MN, Dittus PJ, Kanouse DE, Wallander JL, Pasch KE, Franzini L, Taylor WC, Qureshi T, Franklin FA, Schuster MA. Parental and Peer Factors Associated with Body Image Discrepancy among Fifth-Grade Boys and Girls. J Youth Adolesc 2014; 43(1):15-29.

15. Harrison ME, Norris ML, Obeid N, Fu M, Weinstangel H, Sampson M. Systematic review of the effects of family meal frequency on psychosocial outcomes in youth. Can Fam Physician 2015; 61(2):96-106.

16. Loth K, Wall M, Choi CW, Bucchianeri M, Quick V, Larson N, Neumark-Sztainer D. Family meals and disordered eating in adolescents: are the benefits the same for everyone? Int J Eat Disord 2015; 48(1):100110.

17. Oliveira MM, Campos MO, Andreazzi MAR, Malta DC. Características da Pesquisa Nacional de Saúde do Escolar - PeNSE. Epidemiol. Serv. Saúde 2017; 26(3):605-616.

18. Instituto Brasileiro de Geografia e Estatística (IBGE). Pesquisa Nacional de Saúde do Escolar 2015: Nota metodológica no1: Informações para utilização dos microdados amostras 1 e 2. Rio de Janeiro: IBGE; 2018.

19. Instituto Brasileiro de Geografia e Estatística (IBGE). Pesquisa Nacional de Saúde do Escolar 2009. Rio de Janeiro: IBGE; 2009. (Caderno PeNSE 2009).

20. Instituto Brasileiro de Geografia e Estatística (IBGE). Pesquisa Nacional de Saúde do Escolar 2012. Rio de Janeiro, 2013. (Caderno PeNSE 2012).

21. Instituto Brasileiro de Geografia e Estatística (IBGE). Pesquisa Nacional de Saúde do Escolar 2015. Rio de Janeiro: IBGE; 2016. (Caderno PeNSE 2015).

22. López-Guimerà G, Neumark-Sztainer D, Hannan P, Fauquet J, Loth K, Sánchez-Carracedo D. Unhealthy weight-control behaviours, dieting and weight status: a cross-cultural comparison between North American and Spanish adolescents. Eur Eat Disord Rev 2013; 21(4):276-283.
23. Neumark-Sztainer D, Wall M, Larson NI, Eisenberg ME, Loth K. Dieting and disordered eating behaviors from adolescence to young adulthood: findings from a 10-year longitudinal study. J Am Diet Assoc 2011; 111(7):1004-1011.

24. Youngblade LM, Theokas C, Schulenberg J, Curry L, Huang I-C, Novack M. Risk and promotive factors in families, schools, and communities: A contextual model of positive youth development in adolescence. Pediatrics 2007; 119(Supl.):S47-S53.

25. Walton K, Horton NJ, Rifas-Shiman SL, Field AE, Austin SB, Haycraft E, Breen A, Haines J. Exploring the Role of Family Functioning in the Association Between Frequency of Family Dinners and Dietary Intake Among Adolescents and Young Adults. JAMA Netw Open 2018; 1(7):e185217.

26. Wang ML, Peterson KE, Richmond TK, Spadano-Gasbarro J, Greaney ML, Mezgebu S, McCormick M, Austin SB. Family physical activity and meal practices associated with disordered weight control behaviors in a multiethnic sample of middle-school youth. Acad Pediatr 2013; 13(4):379-385.

27. Vander Wal JS. The relationship between body mass index and unhealthy weight control behaviors among adolescents: the role of family and peer social support. Econ Hum Biol 2012; 10(4):395-404.

28. Neumark-Sztainer D, Hannan PJ, Story M, Croll J, Perry C. Family meal patterns: associations with sociodemographic characteristics and improved dietary intake among adolescents. J Am Diet Assoc 2003; 103(3):317-322.

29. Scaglioni S, De Cosmi V, Ciappolino V, Parazzini F, Brambilla P, Agostoni C. Factors Influencing Children's Eating Behaviours. Nutrients 2018; 10(6).

30. Yee AZ, Lwin MO, Ho SS. The influence of parental practices on child promotive and preventive food consumption behaviors: a systematic review and meta-analysis. Int J Behav Nutr Phys Act 2017; 14(1):47.

31. Almenara CA, Fauquet J, López-Guimerà G, Pamias Massana M, Sánchez-Carracedo D. Self-perceived weight status, dieting, and unhealthy weight-control behaviors among Spanish male adolescents. Nutr Hosp 2014; 30(2):301-305.

32. Tshililo RA, Netshikweta LM, Tshitangano GT, Nemathaga HL. Factors influencing weight control practices amongst the adolescent girls in Vhembe District of Limpopo Province, South Africa. Afr J Prim Health Care Fam Med 2016; 8(2):1-4.

33. Mello FCM, Malta DC, Santos MG, Silva MMA, Silva MAI. Evolução do relato de sofrer bullying entre escolares brasileiros: Pesquisa Nacional de Saúde do Escolar - 2009 a 2015. Rev. bras. epidemiol. 2018; 21 (Supl. 1):e180015.

Artigo apresentado em 30/05/2019

Aprovado em 07/08/2019

Versão final apresentada em 21/11/2019 